\title{
The Use of Longitudinal Mediation Models for Testing Causal Effects and Measuring Direct and Indirect Effects
}

\author{
Paloma Bernal Turnes \\ George Washington University, Washington, D.C., United States; Rey Juan Carlos University, Madrid, Spain \\ Ricardo Ernst \\ Georgetown University, Washington D.C., United States
}

\begin{abstract}
Mediation variables are those intervening variables $(M)$ that affect the relationship between the independent variable $(X)$ and the dependent variable $(Y)$. The causal relation between $X$ and $Y$ through the mediation variable $M$ must be established to analyze mediation. $M$ is a mediator, if it involves indirect effects among the set of original variables $X$ and $Y$. A requirement for a variable to have a causal effect on another is that the cause must precede the outcome in time. This paper argues that the right way to prove the mediation effect is by analyzing different moments in time to support the relations among the variables, which can be done using longitudinal models. The use of traditional analysis in mediation models without the time effect (assumed to occur instantaneously) biases the parameter estimation and could create the possible cancelation of effects, if the paths have opposite signs. Mediation models (without the time effect) are quite frequently used in social sciences. In contrast, the longitudinal mediation model is a very uncommon methodology within the social science community. The aim of this paper is to shed some light about how to analyze the time framework and to provide a methodology to solve the possible limitations that could hinder the robustness of the mediation analysis.
\end{abstract}

Keywords: multi-mediation, longitudinal analysis, time framework, time intervals, latent growth curve model

\section{Mediation Effects in Longitudinal Models}

Mediators are variables through which the influence of an antecedent variable is transferred to a criterion (Mathieu \& Taylor, 2007). The logical ordering of the independent variable $(X)$, the mediatior/s ( $M A$ and/or $M B$, in case multi-mediator variables), and the independent variable $(Y)$ has to be established to analyze mediation (Preacher \& Hayes, 2008).

It means that the indirect effect is only considered as a mediator, when it involves changes in the relations among a set of variables. In other words, by testing the mediation effect, authors are looking for the evidence of causing change in the mediator and/or outcome variables (Zang, Zyphur, \& Preacher, 2009) (see Figure 1). As stated above, a requirement for a variable to have caused another is that the cause must precede the outcome in time (Cole \& Maxwell, 2003; Gollob \& Reichardt, 1987). The way to prove the mediation effect is to analyze

Paloma Bernal Turnes, visiting researcher at George Washington University, Washington, D.C., USA; tenure professor at Rey Juan Carlos University, Madrid, Spain.

Ricardo Ernst, professor of operations and global logistics, Georgetown University, Washington, D.C., USA.

Correspondence concerning this paper should be addressed to Paloma Bernal Turnes, Georgetown University, 3700 O St. NW, 431 Rafik B. Hariri Building, Washington, D.C., USA. 
different moments in time to support the relations among the variables, using longitudinal models (Cole \& Maxwell, 2003; Little, 2013; Maxwell \& Cole, 2007; Selig \& Preacher, 2009). Main limitations of cross-sectional models to analyze causal and mediation effects are overcome by longitudinal models (Cole \& Maxwell, 2003; Cheong, MacKinnon, \& Khoo, 2003; Gollob \& Reichardt, 1987; Maxwell \& Cole, 2007; MacKinnon, Coxe, \& Baraldi, 2012; Selig \& Preacher, 2009). In summary, cross-sectional models describe relations among variables that occur instantaneously and this consideration biases the parameter estimation (Maxwell \& Cole, 2007). However, to describe causality, the previous cause of an ulterior effect requires a gap of time to exert these effects. Longitudinal models suitably model longitudinal data in which the variables are measured in a set of at least two different moments (Cole \& Maxwell, 2003; Gollob \& Reichardt, 1987; Judd \& Kenny, 1981; Kraemer, Wilson, Fairburn, \& Agras, 2002; Selig \& Preacher, 2009; Sobel, 1990).

The longitudinal multi-mediation models address the questions of: Which variable or variables initiate the chain of effects to the dependent variable/s? How does a process of effects cause future outcomes? What is the sequence of effects that influence one another? Additionally, with the analysis of mediation effects and the patterns of those effects over time, it is also possible to use longitudinal models to analyze inter-individual differences (Selig \& Preacher, 2009).

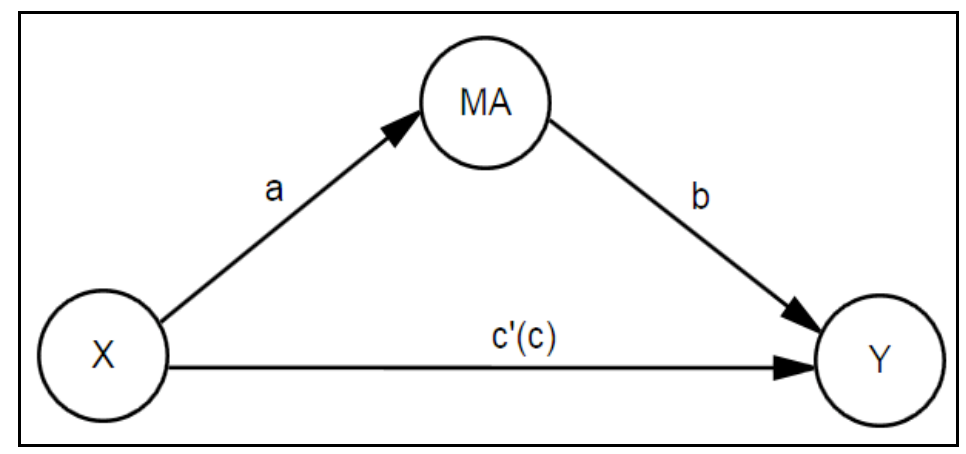

Figure 1. Simple mediator model.

\section{The Effects of Time Over the Variables}

This article focused on the individual differences over time with mediational variables (Cole \& Maxwell, 2003). The analysis over time of the three characteristics that form "the theory of change": stability, stationarity, and equilibrium (Collins, 2006), allows scientists to decide which type of longitudinal model to use (Cole \& Maxwell, 2003; Kenny, 1979; Little, Preacher, Selig, \& Card, 2007; Sobel, 1990): the cross-lagged panel model, the latent growth curve model, or the latent difference score.

Stability refers to the unchanging level of the variable over time (Cole \& Maxwell, 2003; Kenny, 1979). A variable is stable, when its individual values remain significantly "invariable" over time. For instance, in the longitudinal model with mediators (Figure 2), the values of $X_{1}, X_{2}$, and $X_{3}$ are similar over time, in the sense that, at the individual level, the data change similarly among all the samples across time. A case of stability is the bone density, which increases usually until the age of 30 and decreases around age 55 for all individuals. The term "stationary" refers to the similar level of which a variable influences others over time, when it means that the coefficients do not have values significantly different over time - when $a=a^{\prime}=a^{\prime \prime}$, as well as the paths $b, c$, $p, q, r, s$, and $t$ (Cole \& Maxwell, 2003; Kline, 2011; Little et al., 2007). The equilibrium occurs when the correlation between two variables has significantly similar values over time $\left(\sigma x_{1} y_{1}=\sigma x_{2} y_{2}=\ldots=\sigma x_{n} y_{n}\right)$. In 
other words, the linear association between any two variables is in equilibrium, if its correlation has the same value over time or at least converges over time (Cole \& Maxwell, 2003). In a multi-mediator model equilibrium has to be reached as well as the correlation between mediators and other variables. In a model like that in Figure 2, in which MA and MB are the mediators and $X$ is the exogenous variable and $Y$ the endogenous variable, the equilibrium is reached, if the $\sigma x y, \sigma_{X M A}, \sigma_{M A M B}$, and $\sigma_{M B Y}$ are equal or if they converge over time $\left(\sigma x_{1} y_{1}=\sigma x_{2} y_{2}=\ldots=\sigma x_{n} y_{n}, \sigma X_{1} M A_{1}=\sigma X_{2} M A_{2}=\ldots=\sigma X_{n} M A_{n}, \sigma M A_{1} M B_{1}=\sigma M A_{2} M B_{2}=\ldots=\sigma M A_{n} M B_{n}\right.$, and $\left.\sigma M B_{1} y_{1}=\sigma M B_{2} y_{2}=\ldots=\sigma M B_{1} y_{1}\right)$.

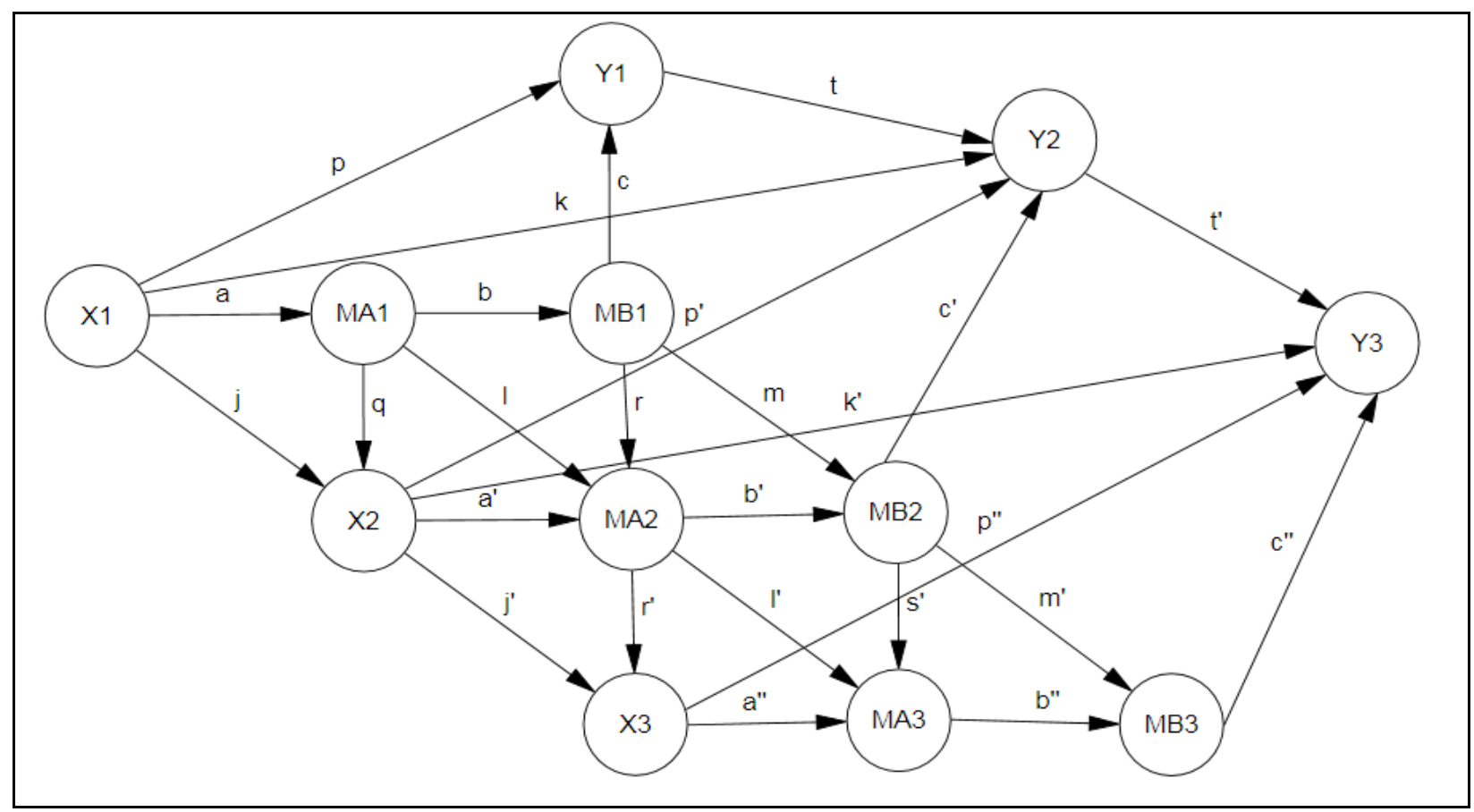

Figure 2. Longitudinal multi-mediation model in three different moments of time. Note: Subscripts denote the time or wave at which the sample was gathered.

\section{The Effects of Time Over in the Research Design}

Time has a considerable impact on the results of any research that is based on several observations of individuals at different moments. The researcher has to observe the speed of changes over time, also has to choose the right period to perform the research and the length of the experiment, and last has to decide the number and frequency of waves to gather data. The decision about the most appropriate time framework has to be based on the goal of the research, the theory (Cole \& Maxwell, 2003), and the specific characteristics of the variables, their causal effects, and individuals. Literature is rich on recommendations about the timing (period), frequency (lag), and spacing (span) to avoid estimation problems in the model or incorrect conclusions (Cole \& Maxwell, 2003; Collins, 2006; Collins \& Graham, 2002; Little, 2013; Little et al., 2007; Selig \& Preacher, 2009) derived by the fact that the size of an effect depends on time interval (Gollob \& Reichardt, 1987).

In longitudinal mediation models, timing becomes a key issue, because the estimation and testing of indirect effects have some difficulties in the assumption of normality and also because there are indirect effects over time (the paths $r$ and $s$ in Figure 2 are examples of indirect effects over time). Collins (2006) and Cole and Maxwell (2003) pointed out that the importance of assessing the timing must be justified scientifically because 
of its implications on results. The literature (Cole \& Maxwell, 2003; Little, 2013; Little et al., 2007) indicates that in order to test the causal relation through mediation effects, just a two-waved model is sufficient for testing mediation hypotheses. Assuming that the optimal lag between each set of variables is equal, authors only needed to test the significance of the causal effects in two different moments of each pair of variables in our model. So then, in the example in Figure 2, it should be possible only to estimate $X_{1} \rightarrow M A_{2}, X_{1} \rightarrow Y_{2}$, $M A_{1} \rightarrow M B_{2}$, and $M B_{1} \rightarrow Y_{2}$ to test the mediation effect. The optimal lag is the time interval of one period for each set of variables, in which the variables are measured in time 1 and time 2 . Once it is tested that the paths $a$, $b, c$, and $d$ are statistically significant different from zero, authors have proved the causal mechanism of change of our structural model. They do it using a longitudinal multi-mediation model by testing ex-ante and ex-post effects.

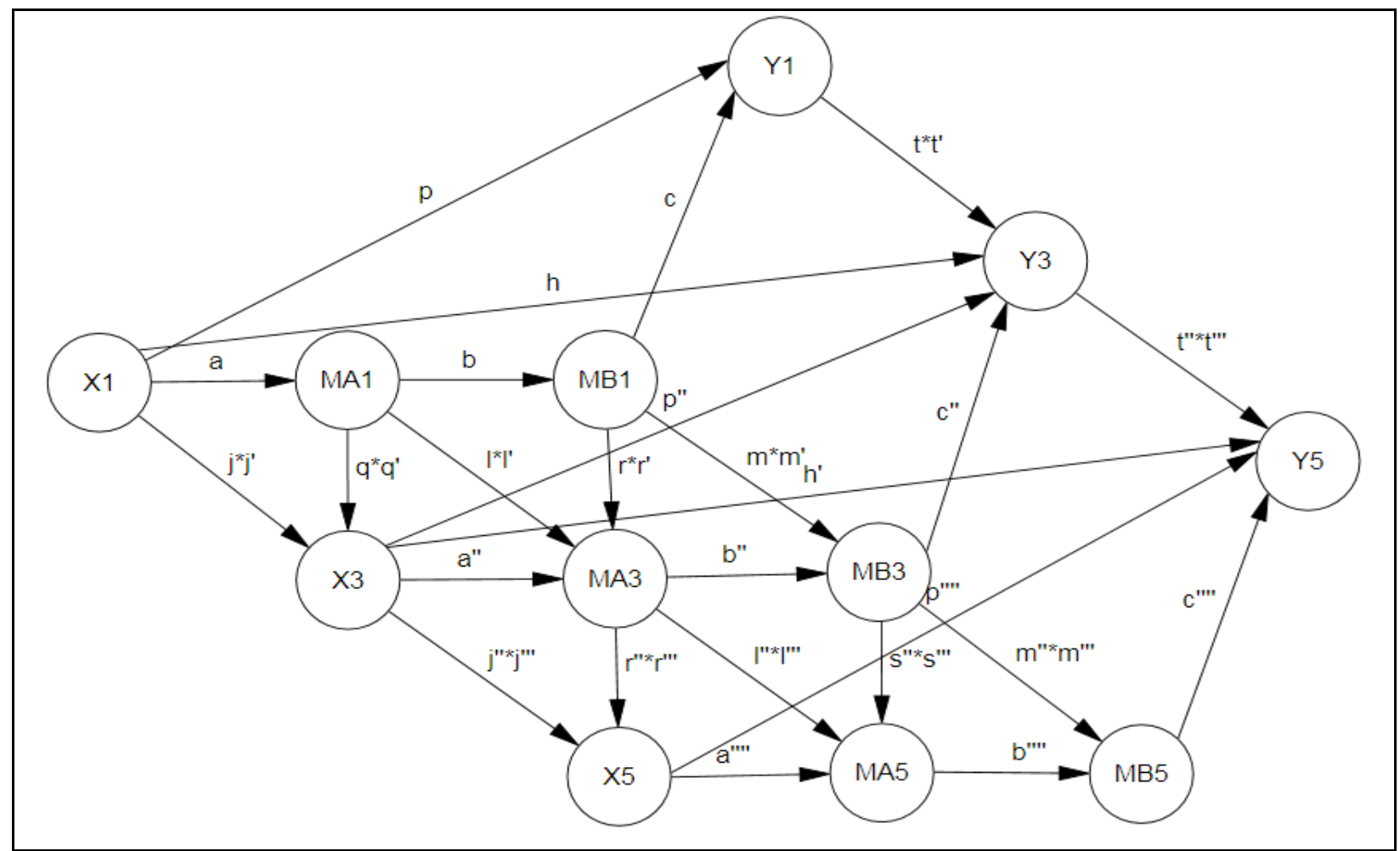

Figure 3. Longitudinal multi-mediation model measured in time interval of two periods. Note: Subscripts denote the time or wave at which the sample was gathered.

In case it is assumed that the optimal lag for the model is the time interval of two periods for each set of variables (e.g., time 1 and time 3), then $h$ path (Figure 3) should be being wrongly estimated as a single direct effect between $X_{1}$ and $Y_{3}$. However, the path $h$ is indeed the sum of all the combined effects (direct and indirect) that are compelled in that two-lag interval between time 3 and time 1 (Figure 2). The combined effects that $X_{1}$ exerts on $Y_{3}$ are actually the sum of all possible paths between the two variables, in which each path is the product of single tracings from the exogenous variable to the endogenous variable in the model of one time interval (Little et al., 2007; Cole \& Maxwell, 2003). In this regard, authors can classify the indirect effect as either time-specific or total (Gollob \& Reichardt, 1991; Selig \& Preacher, 2009). The total effect from the first predictor to the outcome (through mediators) is the sum of all possible time-specific direct and indirect effects that are the alternative paths between the first predictor and the outcome (Cole \& Maxwell, 2003) (Table 1). A 
time-specific effect is calculated by the product of the coefficients which cover the path $h$, that must be calculated following the relationships of the variables (Figure 2). Then, path $h$, instead of just being the result of a single direct effect between $X_{1}$ and $Y_{3}$ as it appears in Figure 4, is instead formed by the sum of the indirect effects in the one-lag model (Table 1), which is:

$$
\begin{aligned}
& h=\left(j \times k^{\prime}\right)+\left(j \times j^{\prime} \times p^{\prime \prime}\right)+\left(a \times b \times r \times r^{\prime} \times p^{\prime \prime}\right)+\left(a \times l \times r^{\prime} \times p "\right)+\left(a \times q \times a^{\prime} \times r^{\prime} \times p^{\prime \prime}\right) \\
& +\left(a \times q \times j^{\prime} \times p^{\prime \prime}\right)+\left(a \times q \times k^{\prime}\right)
\end{aligned}
$$

$$
h=\sum_{i=1}^{T} \alpha^{i-1} \beta^{T-1}
$$

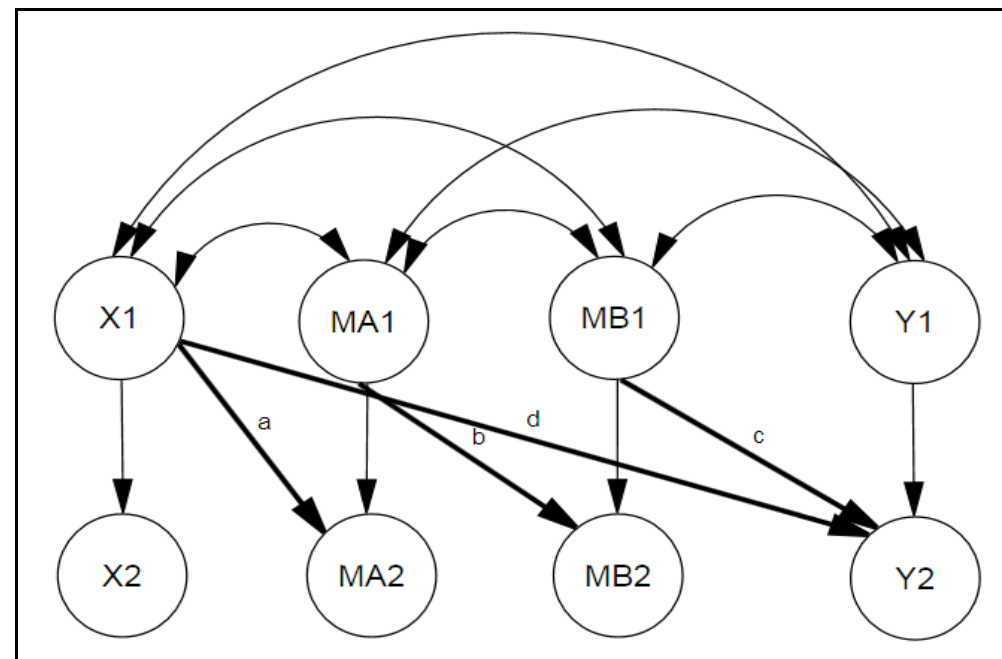

Figure 4. A two-waved longitudinal model for testing multi-mediation effect. Note: Subscripts denote the time or wave at which the sample was gathered.

Table 1

Calculation of Direct, Indirect and Total Effects in Longitudinal Models

\begin{tabular}{llll}
\hline Paths & Effects calculation & Time-specific effects \\
\hline $1 \quad X_{1} \rightarrow X_{2} \rightarrow Y_{3}$ & $j \times k^{\prime}$ & Direct \\
$2 \quad X_{1} \rightarrow X_{2} \rightarrow X_{3} \rightarrow Y_{3}$ & $j \times j^{\prime} \times p^{\prime \prime}$ & Direct \\
$3 \quad X_{1} \rightarrow M A_{1} \rightarrow M B_{1} \rightarrow M A_{2} \rightarrow X_{3} \rightarrow Y_{3}$ & $a \times b \times r \times r^{\prime} \times p^{\prime \prime}$ & Indirect \\
$4 \quad X_{1} \rightarrow M A_{1} \rightarrow M A_{2} \rightarrow X_{3} \rightarrow Y_{3}$ & $a \times l \times r^{\prime} \times p^{\prime \prime}$ & Indirect \\
$5 \quad X_{1} \rightarrow M A_{1} \rightarrow X_{2} \rightarrow M A_{2} \rightarrow X_{3} \rightarrow Y_{3}$ & $a \times q \times a^{\prime} \times r^{\prime} \times p^{\prime \prime}$ & Indirect \\
$6 \quad X_{1} \rightarrow M A_{1} \rightarrow X_{2} \rightarrow X_{3} \rightarrow Y_{3}$ & $a \times q \times j^{\prime} \times p^{\prime \prime}$ & Indirect \\
$7 \quad X_{I} \rightarrow M A_{1} \rightarrow X_{2} \rightarrow Y_{3}$ & $a \times q \times k^{\prime}$ & Indirect \\
Total effects of $X_{1} \rightarrow Y_{3}$ & $h=\Sigma$ & Total effect \\
\hline
\end{tabular}

Finally, as Selig and Preacher (2009) and Cole and Maxwell (2003) stated, reporting the total direct effects and the time-specific indirect effects sheds light in important information about how a process of effects causes future outcomes, instead of merely testing the mediation effects. For the former objective, it should be possible that a three waves model be estimated using two times intervals among the variables of a single mediation model: one time interval for the relationship between the exogenous variable $\left(X_{1}\right)$ and the mediator $\left(M_{2}\right)$, and the other time interval between the mediator $\left(M_{2}\right)$ and the endogenous variable $\left(Y_{3}\right)$. However, the time framework of the experiment must include five periods of collected data to test the trends of effects to cause 
future outcomes. In some cases under scientific justification, the mediator effect could occur in any moment within the time framework of the experiment (Cole \& Maxwell, 2003).

\section{Types of Longitudinal Mediation Models}

The evaluation of longitudinal mediation models is an important step in advancing mediation methods and their characteristics have implications primarily for the estimation process and interpretation. There are three main categories of longitudinal mediation models that arise from changes of the variables over time: the cross-lagged panel model, the latent growth curve model, and the latent difference score.

The cross-lagged panel model reduces the un-biased estimations in the parameters that cross-sectional data cause and allow to assess the changes at inter-individual level (Selig \& Preacher, 2009). However, cross-lagged panel models do not analyze either intra-individual changes or individual differences in intra-individual changes (Selig \& Preacher, 2009). For this reason, it is only recommended to apply this type of models, if the variables do not suffer intra-individual change over time.

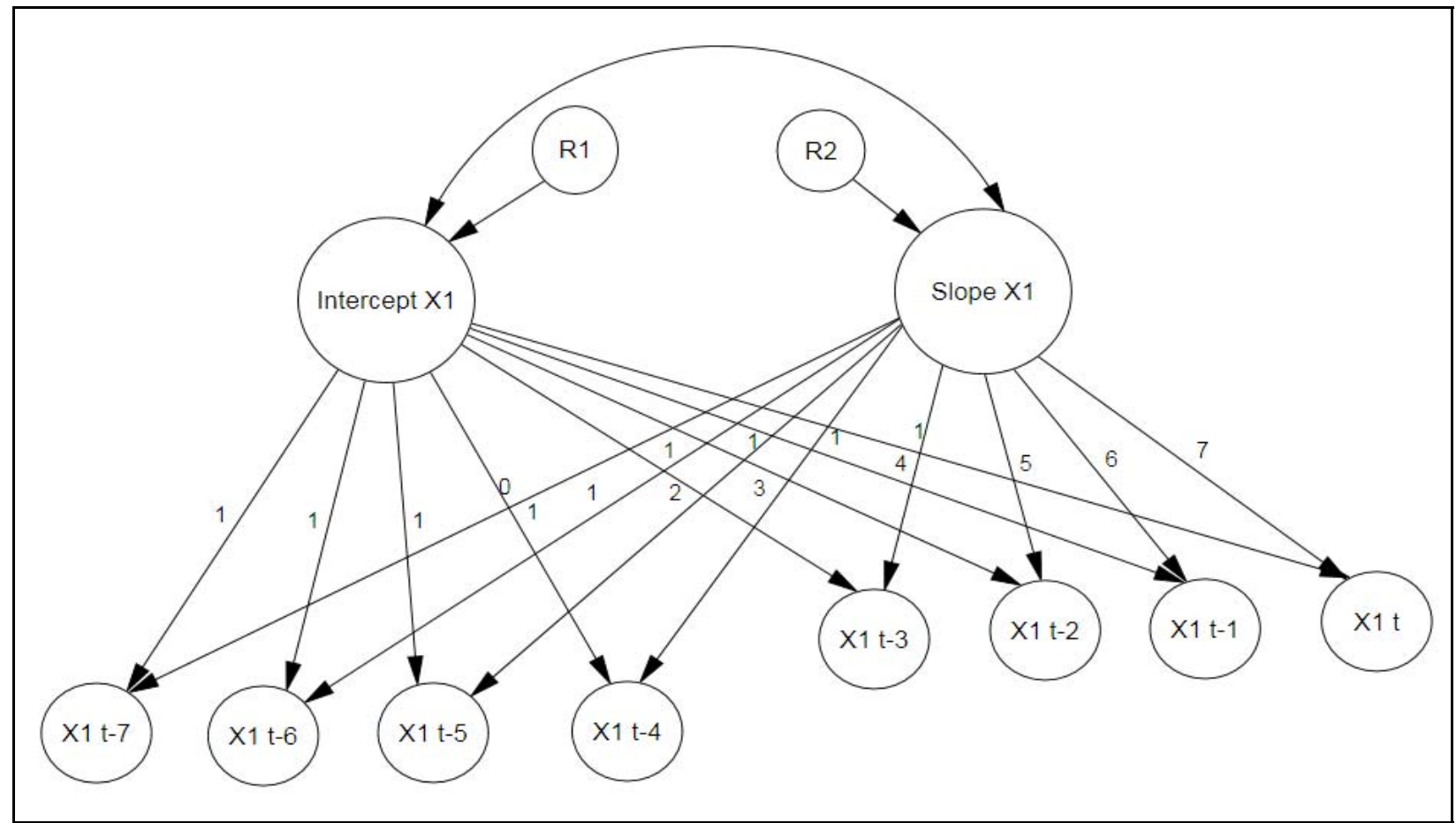

Figure 5. Linear latent growth curve model.

The latent growth curve model is a longitudinal model in which individuals vary significantly over time (Figure 5). The model is formed by latent variables, which are the intercept and the slope of the studying change. Different types of latent growth curve model emerged from the pattern of the growth: linear, quadratic, or exponential (Zhang, Zyphur, \& Preacher, 2012). Techniques for analyzing rates of growth are still relatively rare compared to the evolution of growth curve models, especially those out of the linear growth curve model (Boker \& Nesselroade, 2002; Maxwell \& Cole, 2007; McArdle \& Hamagami, 2001; Ramsay \& Silverman, 2002; Sandland \& McGilchrist, 1979). With latent growth curve model, it is possible to predict the growth trajectory of individuals or variables. As seen in Figure 4, the intercepts, which represent the initial level of growth, are fixed at the value 1 to evaluate all individual growth. The slopes are fixed with a number that shows 
the sequence of time. Because this paper considers eight periods, then the first period of sequence should be $t-$ 7 , and the last period considered should be $t$, which in numbers should become $t-7=0$ and $t=7$ (other values that keep the sequence should be possible too). In latent growth curve model, there are three different types of indirect effects: those involving only intercepts, those involving only slopes, and those that involve intercepts and slopes. As Selig and Preacher (2009) pointed out, when using latent growth curve model, the primary objective is to analyze the intra-individual changes. Then, from the total indirect effects, the indirect effects must be separated from the intercepts.

The third type of longitudinal mediation model is the latent difference score (Figure 6). This kind of model represents different patterns on growth over time, in which patterns change from one interval of time to another. Indeed, the latent difference score is a more complex model based on latent growth curve model, in which the same pattern, either linear, exponential or quadratic, is the same in the full interval period considered. The syntax in Mplus for linear growth models should be the followings (Figure 6):

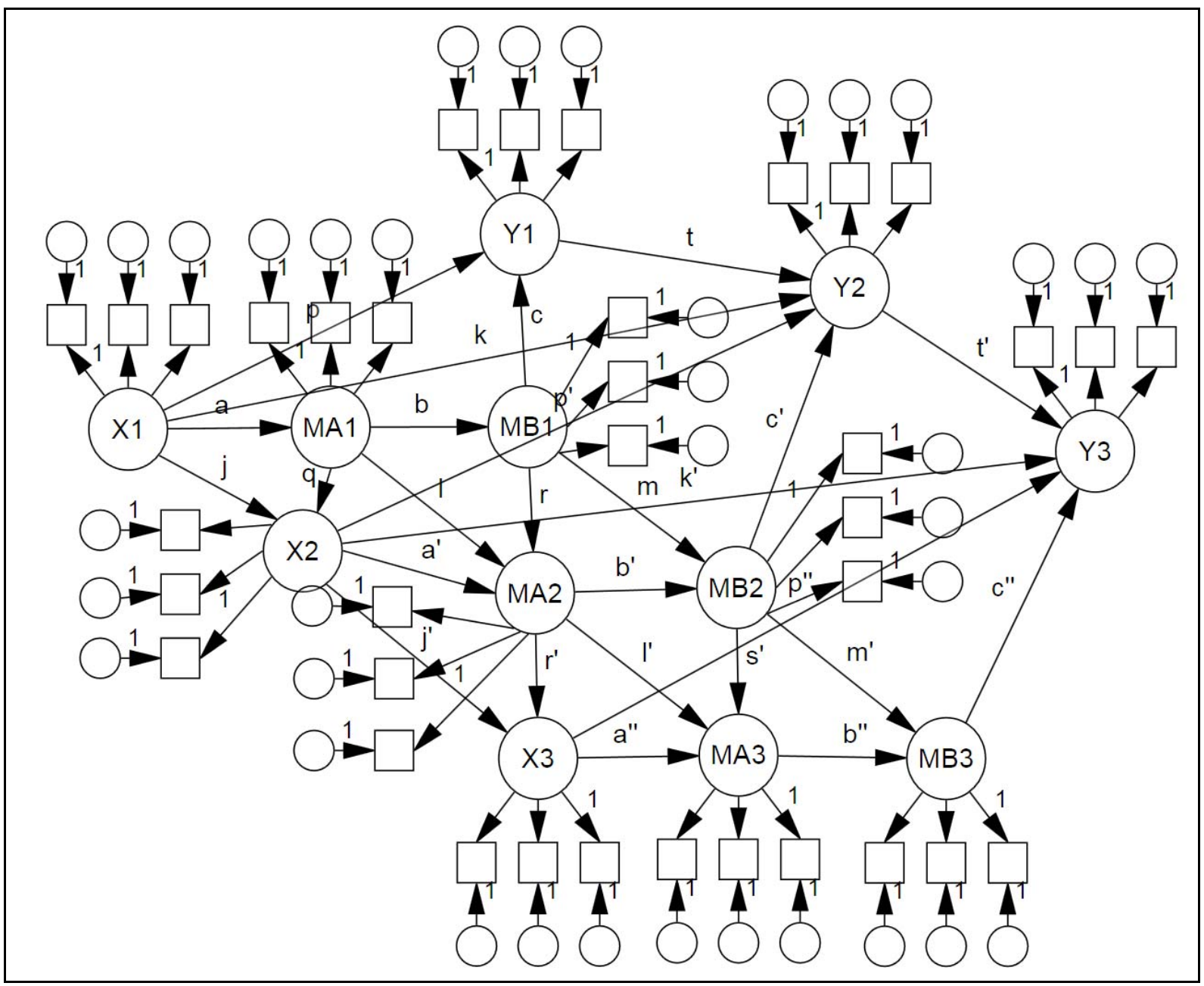

Figure 6. Linear latent growth curve model.

TITLE: Linear latent growth curve model

DATA: FILE IS C:IMy documents $\backslash$ filedata.dat;

VARIABLE: 
NAMES ARE X1_t-7 X1_t-6 X1_t-5 X1_t-4 X1_t -3 X1_t-2 X1_t-1 X1_t;

USEVARIABLES ARE X1_t-7 X1_t-6 X1_t-5 X1_t-4 X1_t-3 X1_t-2 X1_t-1 X1_t; ANALYSIS:

BOOTSTRAP $=10000$;

MODEL: Intercept_X1 BY X1_t-7* X1_t-6 X1_t-5 X1_t-4 X1_t-3 X1_t-2 X1_t-1 X1_t; Intercept_X1@1.0

Slope_X1 BY X1_t-7*@0.0 X1_t-6@1.0 X1_t-5@2.0 X1_t-4@3.0 X1_t-3@4.0 X1_t-2@5.0 X1_t-1@6.0X1_t@7.0;

X1_t-7-X1_t@0 Intercept_X1 Slope_X1;

OUTPUT: TECH1 STANDARDIZED CINTERVAL(BOOTSTRAP);

The syntax in Mplus for linear latent growth model with three indicators per variable should be the followings:

TITLE: Linear latent growth curve model

DATA: FILE IS C:IMy documents $\backslash$ filedata.dat;

VARIABLE:

NAMES ARE

\begin{tabular}{|c|c|c|c|c|c|}
\hline $\mathrm{X} 1 \_\mathrm{t}-2$ & $\mathrm{X} 1 \_\mathrm{t}-1$ & X1_t & $\mathrm{X} 2 \_\mathrm{t}-2$ & X2_t -1 & $\mathrm{X} 2 \_\mathrm{t}$ \\
\hline $\mathrm{X} 3 \_\mathrm{t}-2$ & X3_t -1 & $\mathrm{X} 3 \_\mathrm{t}$ & MA1_t-2 & MA1_t-1 & MA1_t \\
\hline MA2_t -2 & MA2_t-1 & MA2_t & MA3_t-2 & MA3_t-1 & MA3_t \\
\hline MB1_t-2 & MB1_t-1 & MB1_t & MB2_t -2 & MB2_t-1 & MB2_t \\
\hline MB3_t-2 & MB3_t-1 & MB3_t & $\mathrm{Y} 1 \_\mathrm{t}-2$ & $\mathrm{Y} 1 \_\mathrm{t}-1$ & Y1_t \\
\hline $\mathrm{Y} 2 \mathrm{t}-2$ & $\mathrm{Y} 2 \mathrm{t}-1$ & $\mathrm{Y} 2 \mathrm{t}$ & $\mathrm{Y} 3 \mathrm{t}-2$ & Y3 t -1 & Y3 t; \\
\hline
\end{tabular}

USEVARIABLES ARE

\begin{tabular}{|c|c|c|c|c|c|}
\hline $\mathrm{X} 1 \_\mathrm{t}-2$ & $\mathrm{X} 1 \_\mathrm{t}-1$ & $\mathrm{X} 1 \_\mathrm{t}$ & $\mathrm{X} 2 \_\mathrm{t}-2$ & $\mathrm{X} 2 \mathrm{t}-1$ & $\mathrm{X} 2 \_\mathrm{t}$ \\
\hline $\mathrm{X} 3 \_\mathrm{t}-2$ & X3_t -1 & $\mathrm{X} 3 \_\mathrm{t}$ & MA1_t-2 & MA1_t-1 & MA1_t \\
\hline MA2_t -2 & MA2_t-1 & MA2_t & MA3_t-2 & MA3_t-1 & $\mathrm{MA} 3 \_\mathrm{t}$ \\
\hline MB1_t -2 & MB1_t -1 & MB1_t & MB2_t-2 & MB2_t-1 & MB2_t \\
\hline MB3_t-2 & MB3_t-1 & MB3_t & $\mathrm{Y} 1 \_\mathrm{t}-2$ & Y1_t-1 & $\mathrm{Y} 1 \_\mathrm{t}$ \\
\hline$Y 2 \_t-2$ & $Y 2 \_t-1$ & $\mathrm{Y} 2 \_\mathrm{t}$ & $Y 3-t-2$ & $3 \_t-1$ & Y3_t; \\
\hline
\end{tabular}

ANALYSIS: BOOTSTRAP $=10000$;

MODEL:

$\begin{array}{lllll}\text { X1 } & \text { Y } & \text { X1_t }-2 & \text { X1_t }-1 & \text { X1_t; } \\ \text { X2 } & \text { BY } & \text { X2_t }-2 & \text { X2_t }-1 & \text { X2_t; } \\ \text { X3 } & \text { BY } & \text { X3_t-2 } & \text { X3_t }-1 & \text { X3_t; } \\ \text { MA1 } & \text { BY } & \text { MA1_t-2 } & \text { MA1_t-1 } & \text { MA1_t; } \\ \text { MA2 } & \text { BY } & \text { MA2_t-2 } & \text { MA2_t-1 } & \text { MA2_t; } \\ \text { MA3 } & \text { BY } & \text { MA3_t-2 } & \text { MA3_t-1 } & \text { MA3_t; } \\ \text { MB1 } & \text { BY } & \text { MB1_t-2 } & \text { MB1_t-1 } & \text { MB1_t; } \\ \text { MB2 } & \text { BY } & \text { MB2_t-2 } & \text { MB2_t-1 } & \text { MB2_t; } \\ \text { MB3 } & \text { BY } & \text { MB3_t-2 } & \text { MB3_t-1 } & \text { MB3_t; }\end{array}$




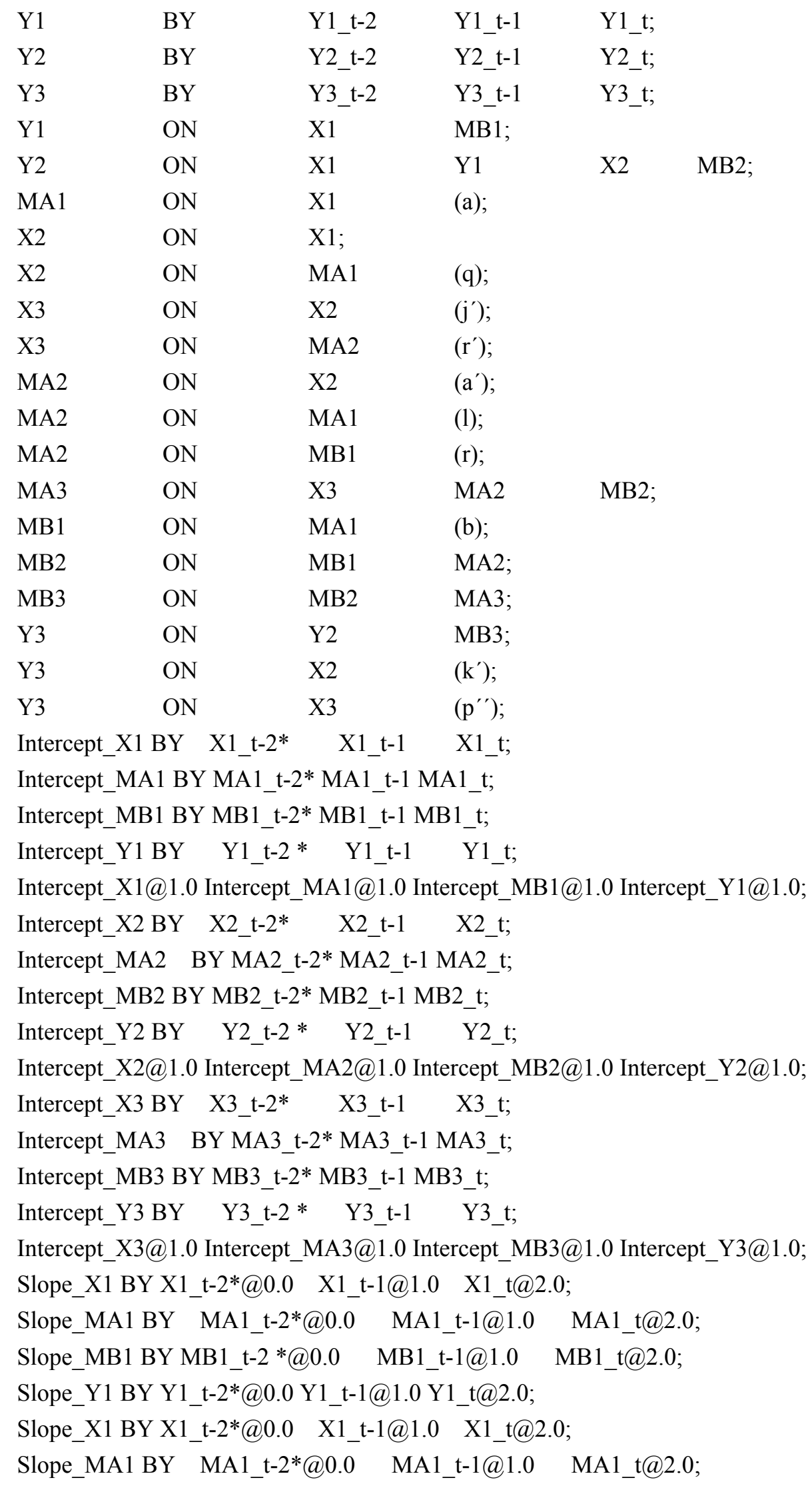




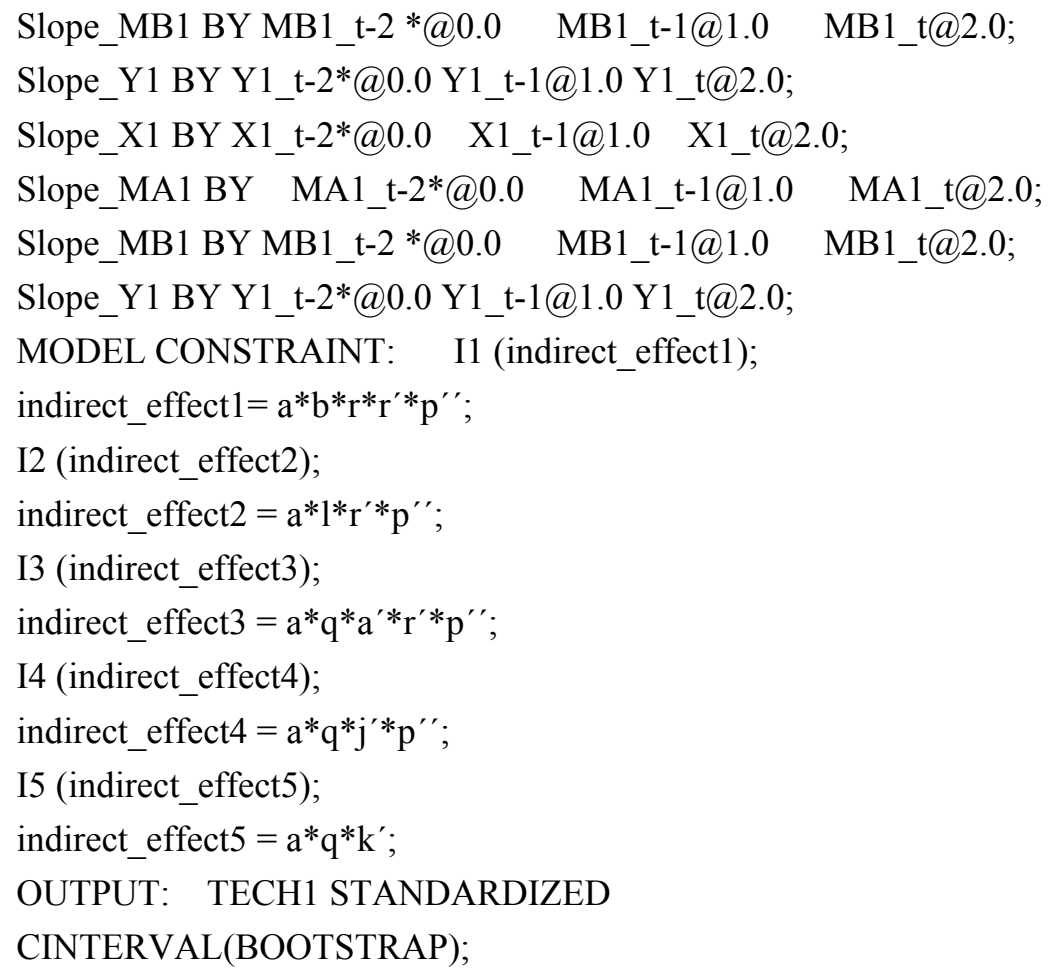

\section{Recommended Steps for Testing Multi-mediation Models}

To analyze multi-mediation models, authors recommended to conduct a longitudinal analysis in order to probe the sequence for how the variables occur and to establish mediation. However, scientists very often do not test if mediation implies a lag of time between the independent/s and dependent variable. This aspect is important, because the lag of time is needed to know that the mediator is acting on the effect that $X$ has on $Y$ (Cole \& Maxwell 2003; Little et al., 2007). By estimating the three regressions, Baron and Kenny (1986) recommended to test mediation as follows:

$$
\begin{gathered}
M=\alpha_{0}+\alpha_{1} X+\varepsilon \\
Y=\gamma_{0}+\gamma_{1} X+\varepsilon \\
Y=\beta_{0}+\beta_{1} X+\beta_{2} M+\varepsilon
\end{gathered}
$$

where $\beta_{1}=b, \alpha_{1}=a$, and $\beta_{2}=c^{\prime}(c)$ and $a, b, c^{\prime}(c)$, and $c$ are unstandardized regression coefficients, generate the limitations of describing relations among variables that occur instantaneously, which biased the parameter estimation in longitudinal models (Maxwell \& Cole, 2007), plus the possible cancelation of effects, if the paths have opposite signs (Zhao, Lynch, \& Chen, 2010). Additionally, to describe causality, the previous cause of an ulterior effect requires a gap of time to exert these effects, which requires to measure the variables in a set of at least two different moments (Cole \& Maxwell, 2003; Gollob \& Reichardt, 1987; Judd \& Kenny, 1981; Kraemer et al., 2002; Selig \& Preacher, 2009; Sobel, 1990). For these reasons, authors suggested to conduct a deeper analysis to test mediation models. First, the conceptual model with multi-mediation variables must be estimated with bootstrapping, because this technique does not impose normal distribution to the variables. In mediation models, they estimated the product of variables, which are not normally distributed. Bootstrapping reports the confident interval in which the parameter estimated is significant, if this confident interval excludes the zero value between its upper and lower bounds. Second, the analysis of the mediation effects must be 
proceeded. Researchers must establish the adequate data intervals to test the sequence of causal effects over time. Once the optimal lag is determined, they suggested using the simplest analysis over time. This is the one that considers two different consecutive moments: time one and time two (Figure 4), but a wider interval of more time periods is also valid. In the example of two mediators, authors will additionally test the model drawn in Figure 2, which involves testing the significance of the coefficients $\alpha_{1}, \gamma_{1}, \delta_{1}$, and $\beta_{1}$ of the following regressions extracted from that model:

$$
\begin{gathered}
M A 2=\alpha_{0}+\alpha_{1} X 1+\varepsilon \\
Y 2=\gamma_{0}+\gamma_{1} X 1+\varepsilon \\
M B 2=\delta_{0}+\delta_{1} M A 1+\varepsilon \\
Y 2=\beta_{0}+\beta_{1} M B 1+\varepsilon
\end{gathered}
$$

where $\alpha_{1}=a, \gamma_{1}=d, \delta_{1}=b$, and $\beta_{1}=c$ in Figure 6 .

If the coefficients $\alpha_{1}, \gamma_{1}, \delta_{1}$, and $\beta_{1}$ are statistically significant, authors have tested that the causal effects occur following the next sequence: $X \rightarrow M A \rightarrow M B \rightarrow Y$. In this step in which the sequence is tested, the research must incorporate the time relationships in the conceptual model. This is, indeed, an invitation to specify a longitudinal mediation model. Researchers must compare the improvements in accuracy, if any, with the longitudinal mediation model on the conceptual model. Third, authors will calculate and report the total effects, the direct, and the indirect effects providing the information of the time-specific effects in case of applying longitudinal multi-mediation models with the signal of the effects. In the multi-mediation model in Figure 6, if $a \times b \times d \times c^{\prime}(c)$ is not significant, there are only direct effects between $X$ and $Y$, which means that there are not mediation effects between $X$ and $Y$. In contrast, if $c^{\prime}(c)$ is not significant, there are only indirect effects between $X$ and $Y$, and the global amount of those indirect effects should be positive or negative. However, it is controversial to analyze indirect effects with opposite signs. Baron and Kenny (1986) argued that the signal of the product is the signal of the indirect effect. However, other authors (Collins, Graham, \& Flaherty, 1998; MacKinnon, Krull, \& Lockwood, 2010; Shrout \& Bolger, 2002) considered that in some situations, the addition of the opposite signs effect determines better the global sign of the indirect effect. In contrast, authors argued that the global sign of the indirect effect is a matter of signal and magnitude, realizing that the final sign is difficult to determine (Zhao et al., 2010), especially when a model uses Likert scales in its variables.

\section{Conclusions}

The use of mediation models appears very often in the social sciences literature. Instead of analyzing theoretical and empirical evidence of mediation, the causal effects are too often only theoretically demonstrated. It is usually explained by the requirement that a variable causes another, assuming that the cause must precede the outcome over time. Thus, a longitudinal analysis must be developed to test the pattern and sequence of effects in mediational models.

Authors have discovered that the longitudinal mediation model is a very uncommon methodology within the social sciences community. Just reviewing the papers published in the three top indexed journals in the business arena during 2012 and 2013 shows that within the papers that use mediation variables, only $1 \%$ use longitudinal models. In this paper, they proposed the use of longitudinal methodologies with mediation to overcome the limitations of existing models which only rely on mediation (Cole \& Maxwell, 2003; Collins, 2006; Collins \& Graham, 2002; Gollob \& Reichardt, 1987; Little, 2013; Little et al., 2007; Selig \& Preacher, 
2009). The longitudinal analysis makes existing models more accurate, for example, bootstrap test is more rigorous than product coefficient approaches, with the Sobel test (1990) among others, but neither of these test the sequence of effects among variables. This paper presents a tutorial which should correct this deficit. Authors added to the modern literature of mediation and the characteristics of multi-mediation effects in longitudinal models, by presenting a methodology to conduct the longitudinal analysis, providing an example of syntax to conduct the analysis, and interpreting the type of direct and indirect effects that merge using time lags in the sample. Future research could address the question of mediation effects with opposite signs and its interpretation. Authors acknowledged that there is a lack of literature that reflects the difficulties of interpreting opposite signs of total effects when mixing Likert scales and continuous variables.

\section{References}

Baron, R. M., \& Kenny, D. A. (1986). The moderator-mediator distinction in social psychological research: Conceptual, strategic, and statistical considerations. Journal of Personality and Social Psychology, 51(6), 1173-1182.

Boker, S. M., \& Nesselroade, J. R. (2002). A method for modeling the intrinsic dynamics of intraindividual variability: Recovering the parameters of simulated oscillators in multi-wave data. Multivariate Behavioral Research, 37, 127-160.

Bryk, A. S., \& Raudenbush, S. W. (1992). Hierarchical linear models: Applications and data analysis methods. Newbury Park, CA: Sage.

Cheong, J., MacKinnon, D. P., \& Khoo, S. T. (2003). Investigation of mediational processes using parallel process latent growth curve modeling. Structural Equation Modeling: A Multidisciplinary Journal, 10(2), 238-262.

Cole, D. A., \& Maxwell, S. E. (2003). Testing mediational models with longitudinal data: Questions and tips in the use of structural equation modeling. Journal of Abnormal Psychology, 112(4), 558-577.

Collins, L. M. (2006). Analysis of longitudinal data: The integration of theoretical model, temporal design, and statistical model. Annual Review of Psychology, 57, 505-528.

Collins, L. M., Graham, J. W., \& Flaherty, B. P. (1998). An alternative framework for defining mediation. Multivariate Behavioral Research, 33(2), 295-312.

Gollob, H. F., \& Reichardt, C. S. (1987). Taking account of time lags in causal models. Child Development, 58, 80-92.

Gollob, H. F., \& Reichardt, C. S. (1991). Interpreting and estimating indirect effects assuming time lags really matter. In L. M. Collins and J. L. Horn (Eds.), Best methods for the analysis of change: Recent advances, unanswered questions, future directions (pp. 243-259). Washington, DC: American Psychological Association.

Judd, C. M., \& Kenny, D. A. (1981). Process analysis: Estimating mediation in treatment evaluations. Evaluation Review, 5 , 602-619.

Kraemer, H. C., Wilson, G. T., Fairburn, C. G., \& Agras, W. S. (2002). Mediators and moderators of treatment effects in randomized clinical trials. Archives of General Psychiatry, 59, 877-884.

Little, T. D. (2013). Longitudinal structural equation modeling. New York, NY: The Guilford Press.

Little, T. D., Preacher, K. J., Selig, J. P., \& Card, N. A. (2007). New developments in latent variable panel analyses of longitudinal data. International Journal of Behavioral Development, 31(4), 357-365.

MacKinnon, D. P., Coxe, S., \& Baraldi, A. N. (2012). Guidelines for the investigation of mediating variables in business research. Journal of Business Psychology, 27, 1-14.

MacKinnon, D. P., Krull, J. L., \& Lockwood, C. M. (2000). Equivalence of the mediation, confounding and suppression effect. Prevention Science, 1(4), 173-181.

Mathieu, J. E., \& Taylor, S. R. (2007). A framework for testing meso-mediational relationships in organizational behavior. Journal of Organizational Behavior, 28, 141-172.

Maxwell, S. E., \& Cole, D. A. (2007). Bias in cross-sectional analysis of longitudinal mediation. Psychological Methods, 12, 23-44.

McArdle, J. J., \& Hamagami, F. (2001). Latent difference score structural models for linear dynamic analysis with incomplete longitudinal data. In L. Collins and A. Sayer (Eds.), New methods for the analysis of change (pp. 139-175). Washington, DC: American Psychological Association.

Preacher, K. J., \& Hayes, A. F. (2008). Asymptotic and resampling strategies for assessing and comparing indirect effects in 
multiple mediator models. Behavior Research Methods, 40(3), 879-891.

Ramsay, J. O., \& Silverman, B. W. (2002). Applied functional data analysis: Methods and case studies. Berlin: Springer.

Sandland, R. L., \& McGilchrist, C. A. (1979). Stochastic growth curve analysis. Biometrics, 35, 255-271.

Selig, J. P., \& Preacher, K. J. (2009). Mediation models for longitudinal data in developmental research. Research in Human Development, 6(2-3), 144-164.

Shrout, P. E., \& Bolger, N. (2009). Mediation in experimental and non-experimental studies: New procedures and recommendation. Psychological Methods, 1(4), 422-445.

Sobel, M. E. (1990). Effect analysis and causation in linear structural equation models. Psychometrika, 55, 495-515.

Zang, Z., Zyphur, M. J., \& Preacher, K. J. (2009). Testing multilevel mediation using hierarchical linear models. Problems and solutions. Organizational Research Methods, 12(4), 695-719.

Zhao, X., Lynch, J. G., \& Chen, Q. (2010). Reconsidering Baron and Kenny: Myths and truths about mediation analysis. Journal of Consumer Research, 37(2), 197-206.

Kenny, D. A. (1979). Correlation and causality. New York, NY: John Wiley \& Sons.

Kline, R. B. (2011). Principles and practice of structural equation modeling (3rd ed.). New York: Guilford Press.

Collins, L. M., \& Graham, J. W. (2002). The effect of the timing and spacing of observations in longitudinal studies of tobacco and other drug use: Temporal design considerations. Drug and Alcohol Dependence, 68(1), 85-96. 